\title{
A comparison of measured versus self-reported anthropometrics for assessing obesity in adults : a literature review
}

\section{Maukonen, Mirkka}

2018-07

Maukonen, M , Mannisto , S \& Tolonen , H 2018 , ' A comparison of measured versus self-reported anthropometrics for assessing obesity in adults : a literature review ' , Scandinavian Journal of Public Health, vol. 46 , no. 5 , pp. 565-579 . https://doi.org/10.1177/1403494818761971

http://hdl.handle.net/10138/304269

https://doi.org/10.1177/1403494818761971

unspecified

publishedVersion

Downloaded from Helda, University of Helsinki institutional repository.

This is an electronic reprint of the original article.

This reprint may differ from the original in pagination and typographic detail.

Please cite the original version. 


\title{
A comparison of measured versus self-reported anthropometrics for assessing obesity in adults: a literature review
}

\author{
MIRKKA MAUKONEN ${ }^{1,2}$, SATU MÄNNISTÖ $^{1} \&$ HANNA TOLONEN $^{1}$ \\ ${ }^{1}$ Department of Public Health Solutions, National Institute for Health and Welfare, Helsinki, Finland, and ${ }^{2}$ Department of \\ Public Health, University of Helsinki, Finland
}

\begin{abstract}
Aims: Up-to-date information on the accuracy between different anthropometric data collection methods is vital for the reliability of anthropometric data. A previous review on this matter was conducted a decade ago. Our aim was to conduct a literature review on the accuracy of self-reported height, weight, and body mass index (BMI) against measured values for assessing obesity in adults. To obtain an overview of the present situation, we included studies published after the previous review. Differences according to sex, BMI groups, and continents were also assessed. Methods: Studies published between January 2006 and April 2017 were identified from a literature search on PubMed. Results: Our search retrieved 62 publications on adult populations that showed a tendency for self-reported height to be overestimated and weight to be underestimated when compared with measured values. The findings were similar for both sexes. BMI derived from self-reported height and weight was underestimated; there was a clear tendency for underestimation of overweight (from $1.8 \%$-points to $9.8 \%$-points) and obesity (from $0.7 \%$-points to $13.4 \%$-points) prevalence by self-report. The bias was greater in overweight and obese participants than those of normal weight. Studies conducted in North America showed a greater bias, whereas the bias in Asian studies seemed to be lower than those from other continents. Conclusions: With globally rising obesity rates, accurate estimation of obesity is essential for effective public health policies to support obesity prevention. As self-report bias tends to be higher among overweight and obese individuals, measured anthropometrics provide a more reliable tool for assessing the prevalence of obesity.
\end{abstract}

Keywords: Accuracy, anthropometry, body mass index, body height, body weight, obesity, self-report

\section{Introduction}

Obesity has become one of the main public health challenges worldwide [1]. The prevalence of obesity has more than doubled since the 1980s, and it has been estimated that at least one-third of the world's adult population is either overweight or obese [2]. Obesity is a multifactorial condition that causes metabolic dysregulations that increase the risk of several non-communicable diseases (NCDs), such as cardiovascular disease (CVD) and type 2 diabetes. The World Health Organization (WHO) has set the global targets for the prevention of NCDs; one of these is to stop the increase in obesity [3].
Successful monitoring of overweight and obesity in the general population is necessary for effective public health policy. The WHO recommends that countries conduct health examination or nutrition surveys with anthropometric measures (e.g., height and weight) to evaluate whether the global targets will be reached [3]. In many countries, information on overweight and obesity prevalence is based on self-reported anthropometrics, which are easier and a more time-efficient and cost-efficient data collection method in large population samples than objective measurements. 
Several factors can cause bias on self-reported anthropometric measures and influence the magnitude of the bias, such as sex, age, and body mass index (BMI). These reporting biases may result in inaccurate estimation of BMI and consequently the prevalence of obesity, which leads to an unreliable assessment of the disease and the mortality risk of a population $[4,5]$. A previous review on the validity of self-reported height and weight was published a decade ago in 2007 [6]. This review showed that people overestimated their height and underestimated their weight in a wide range of different populations compared to measured values. In particular, women and participants who were overweight or obese underestimated their weight, whereas men and participants in older age groups overestimated their height. Height overestimation among older age groups is common, as people may report height that had been measured when they were younger and with increasing age people tend to become shorter due to changes in bone and muscle structures.

Increasing health awareness and social desirability, especially in western societies, are important factors that may lead people to report height and weight values that are closer to their ideal rather than to actual values $[7,8]$. At the same time, rising obesity rates may cause heavier body weights to appear more normal and result in the normalization of obesity, which may affect selfreporting bias as well $[9,10]$. Although the bias related to self-reported anthropometrics is well acknowledged, it is not known how these changes have affected the present overall picture of the self-reporting bias. There are few studies that have examined temporal changes in bias of anthropometric measurements based on selfreporting and the results from these studies are conflicting [11-14]. Therefore, to obtain reliable anthropometric data, up-to-date information on the accuracy between different anthropometric data collection methods and up-to-date knowledge about potential bias due to data collection methods is needed.

Our aim was to conduct a literature review of the present situation in self-reported height, weight and BMI validity against measured values for assessing obesity in adults. Differences according to sex, BMI groups, and continents were also assessed. To obtain up-to-date information and an overall picture of the present situation, we included studies published after the systematic review of Connor Gorber et al. [6].

\section{Methods of the review}

\section{Search strategy}

Published studies were identified from a literature search on PubMed. The search of this non-systematic review was restricted to full papers published in
English between January 2006 and April 2017 and to adult populations (age $>18$ ) with sample size $>100$ participants. The search terms included following keywords: (measured weight[Title/Abstract] OR measured height[Title/Abstract] OR measured obesity[Title/ Abstract] OR measured BMI[Title/Abstract] OR obesity measure ${ }^{\star}\left[\right.$ Title/Abstract] OR clinical measure ${ }^{\star}$ [Title/Abstract] OR objective measure [Title/Abstract] OR direct measure ${ }^{\star}\left[\right.$ Title/Abstract]) OR measure ${ }^{\star}$ [Title/Abstract] AND (self report ${ }^{\star}$ [Title]) AND (anthropometry[MeSH Terms] OR body mass index[MeSH Terms] OR body weight[MeSH Terms] OR body height[MeSH Terms] OR height[Title] OR weight[Title] OR obesity[Title]).

\section{Methods}

The results from individual studies are presented as combined data for men and women (overall sample) and according to sex and BMI groups with mean differences (self-reported minus measured values) and standard deviations (SD) of these mean differences. SDs were calculated whenever possible if confidence intervals or standard errors were reported in the original publications. The anthropometrics are given in the international system of units (SI). If other units were used in the original publications, equivalent SI values were calculated. Furthermore, WHO guidelines were followed (unless otherwise noted) in classification of BMI: underweight $\left(<18.5 \mathrm{~kg} / \mathrm{m}^{2}\right)$, normal (18.5-24.9 $\left.\mathrm{kg} / \mathrm{m}^{2}\right)$, overweight (25.0-29.9 $\left.\mathrm{kg} / \mathrm{m}^{2}\right)$, and obese $\left(\geq 30.0 \mathrm{~kg} / \mathrm{m}^{2}\right)$.

\section{Results}

Search

The initial database search plan and selection of the keywords were conducted with an information specialist. MM made the final selection of the keywords, conducted the PubMed search on 3 May 2017, and performed the selection of the publications. If there was uncertainty in decision making, the final decision was made with HT. The total number of publications identified was 415 . After reading the titles and abstracts, an initial selection of 61 publications was made. These were read in full, and finally 58 publications were included in the review. Four additional publications were identified by reviewing the references of these publications; thus the final number of publications in the review was 62 (Table I).

\section{Height}

Overall sample (combined data on men and women). Data on self-reported and measured height 


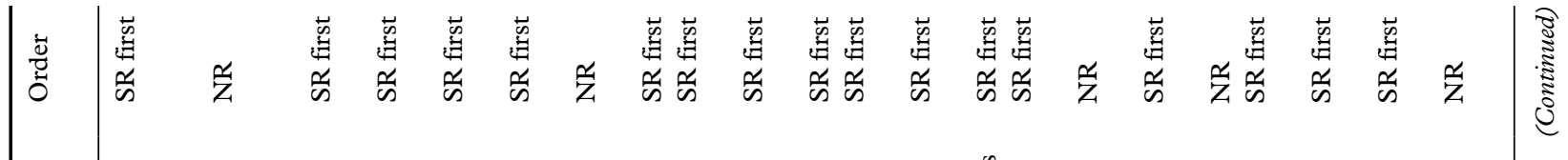

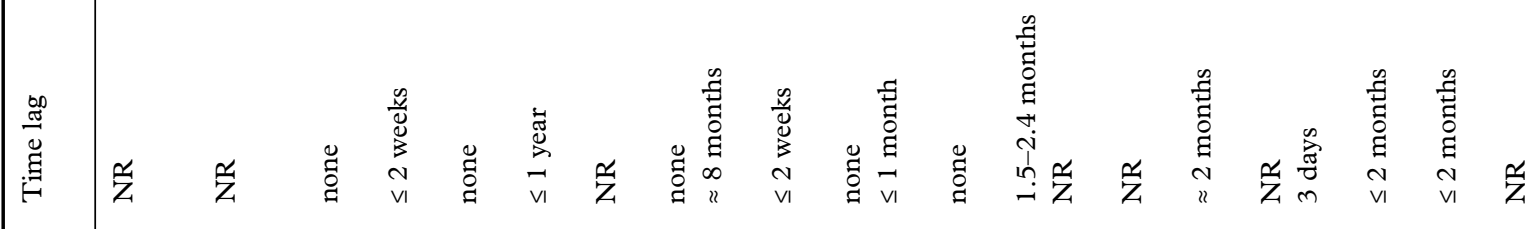

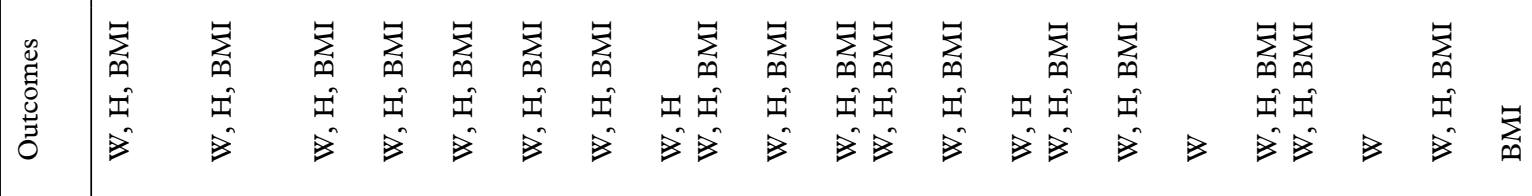

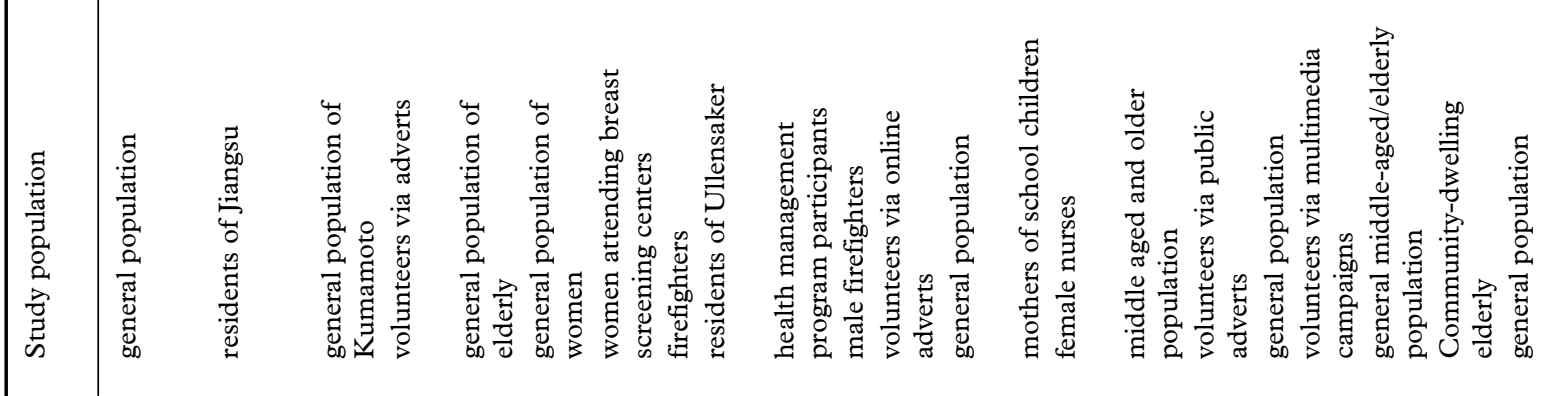

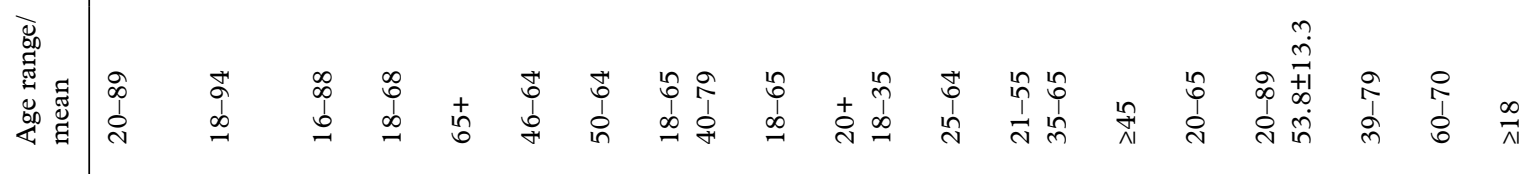

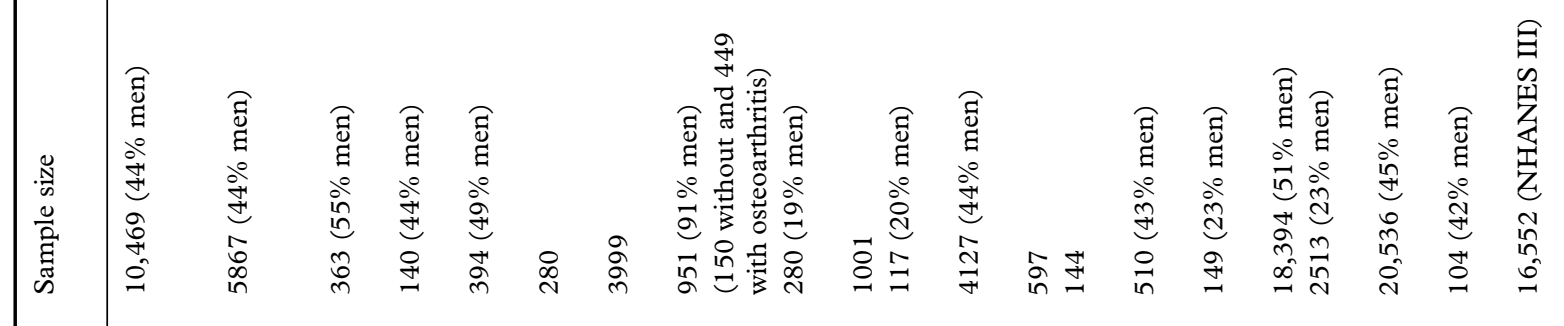

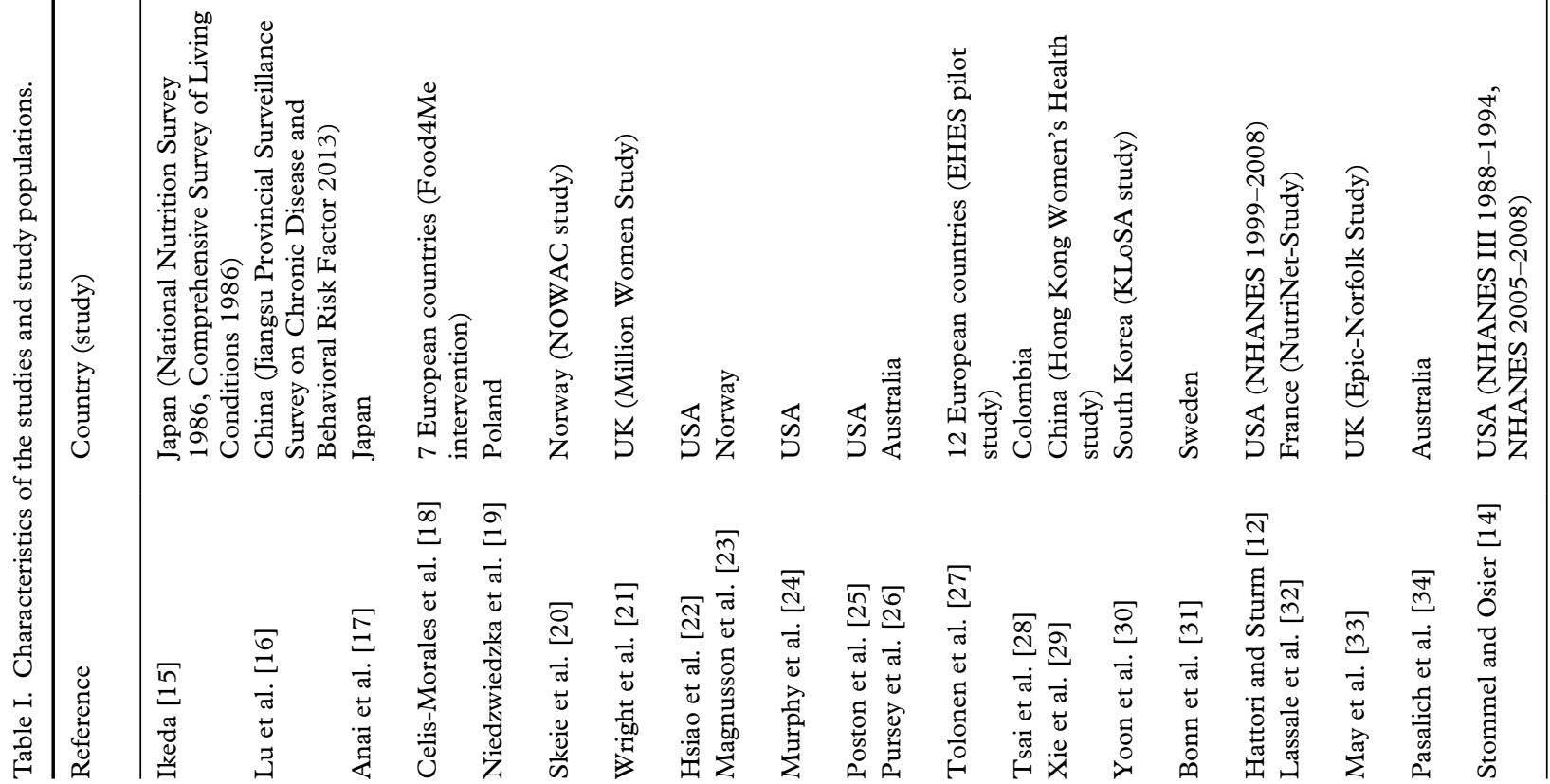




\begin{tabular}{|c|c|c|c|c|c|c|c|c|c|c|c|c|c|c|}
\hline & 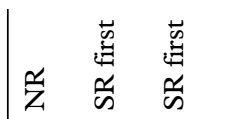 & 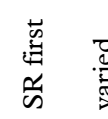 & 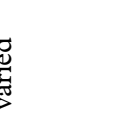 & 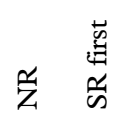 & $\begin{array}{l}\text { 蔷 } \\
\text { 点 }\end{array}$ & 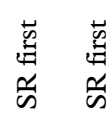 & 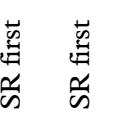 & & & & 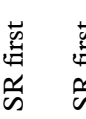 & 蓸 & $\begin{array}{l}\text { 鄫 } \\
\text { 空 }\end{array}$ & \\
\hline & 苂 苂 并 & 畜 & 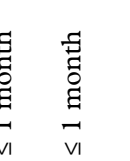 & $\ddot{z}$ & 苾 & 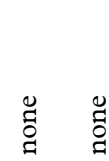 & 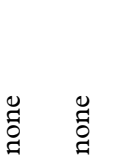 & 苂 & בे & & & & 竒 & 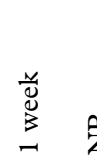 \\
\hline 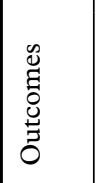 & 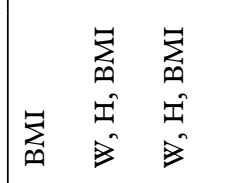 & 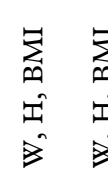 & 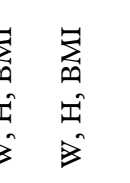 & 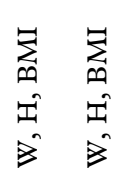 & 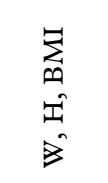 & 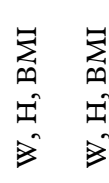 & 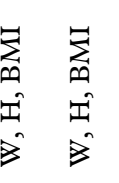 & 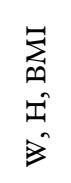 & $\sum_{\text {I }}^{*}$ & & 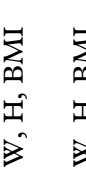 & & 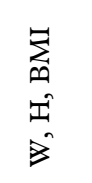 & \\
\hline & 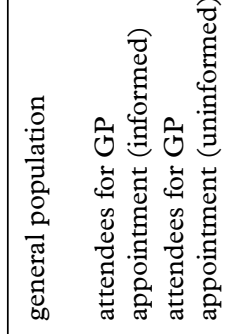 & 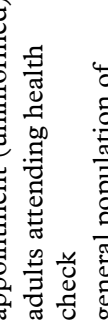 & 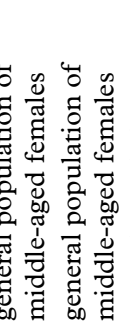 & 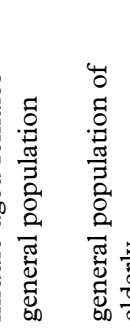 & 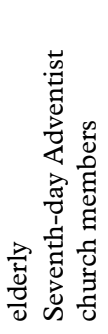 & 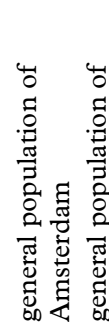 & & 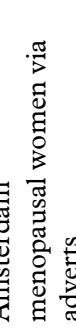 & 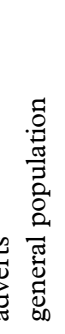 & & 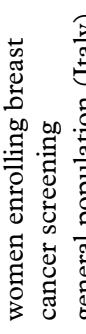 & & 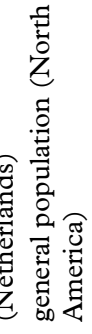 & 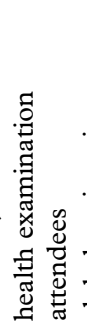 \\
\hline & $\stackrel{+}{\stackrel{ \pm}{ \pm}} \quad \stackrel{+}{\sim}$ & & & 㕝 & 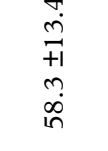 & & 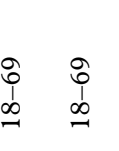 & $\begin{array}{l}0 \\
\vdots \\
\vdots\end{array}$ & $d_{d}^{d}$ & & & & 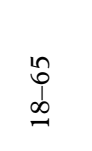 & 㣽 \\
\hline & 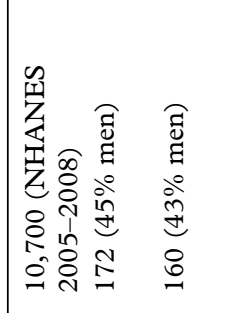 & 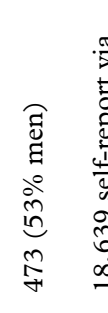 & 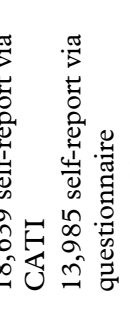 & 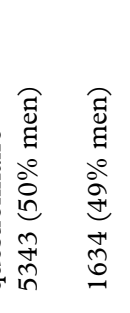 & 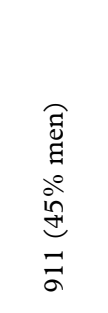 & 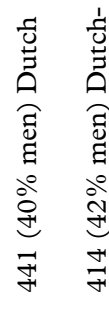 & 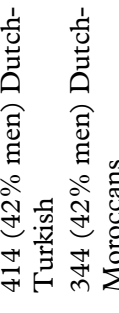 & & 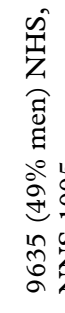 & & 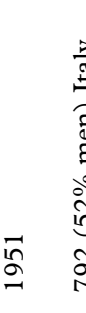 & & 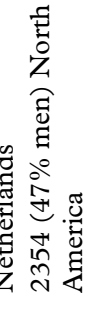 & 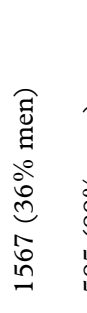 \\
\hline & 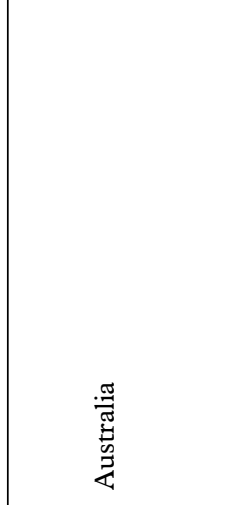 & 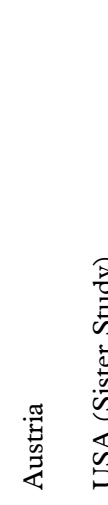 & 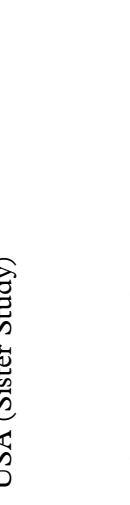 & 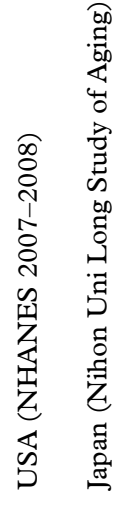 & 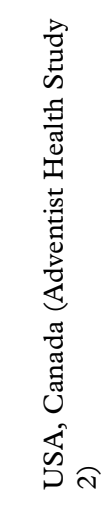 & 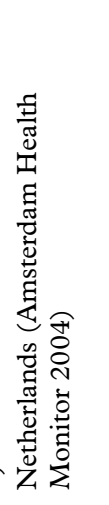 & & 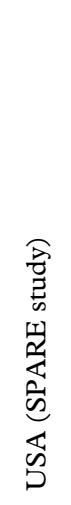 & 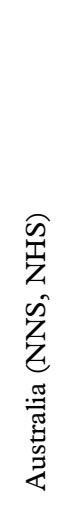 & & & & & 总 \\
\hline & 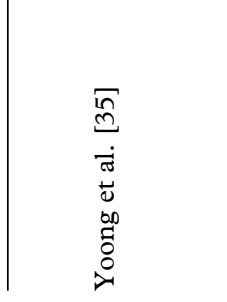 & 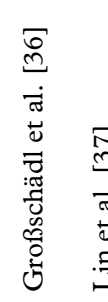 & 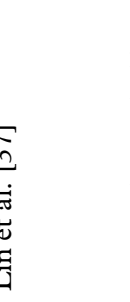 & 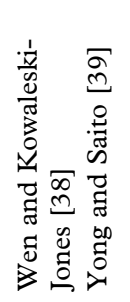 & 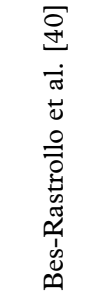 & 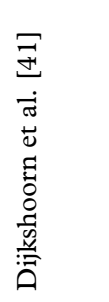 & & 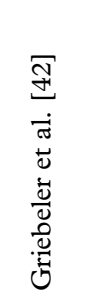 & $\frac{\bar{P}}{7}$ & & 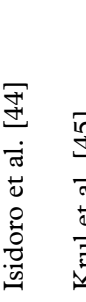 & & & 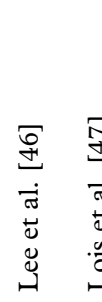 \\
\hline
\end{tabular}




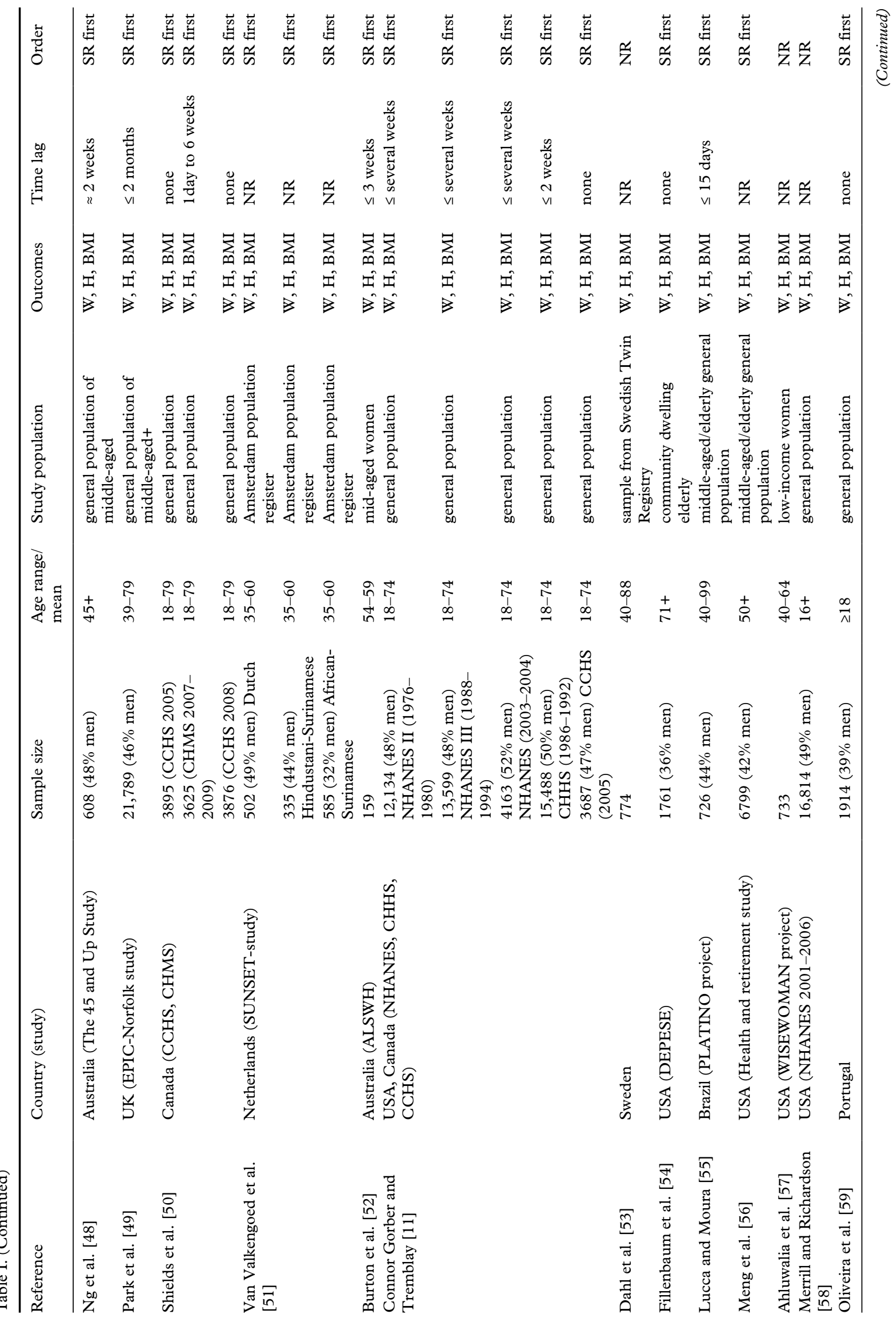




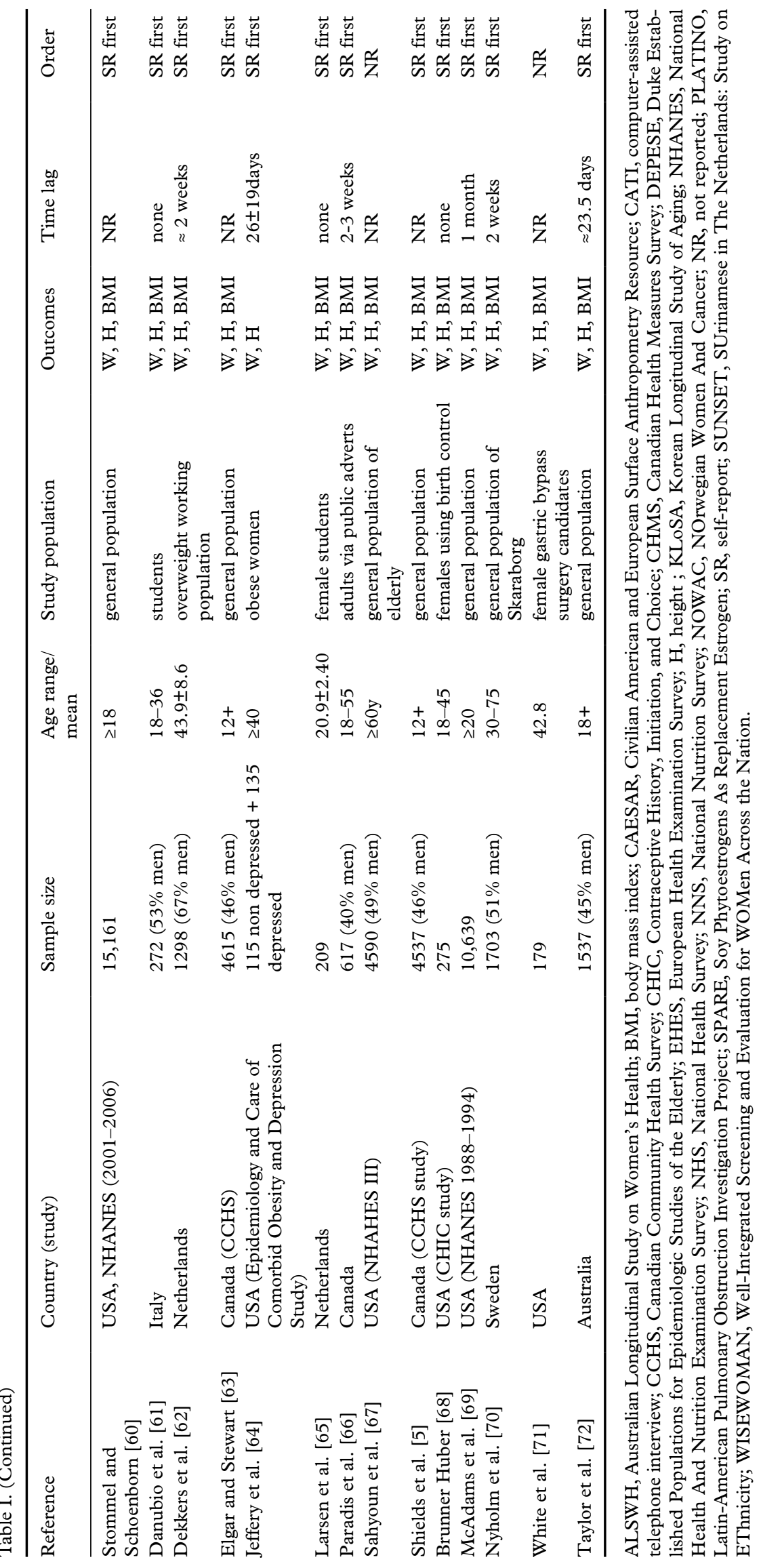


was obtained from 58 studies (see Supplementary Table I online). Of these, 25 studies reported combined data on men and women; the majority showed that height was overestimated by self-report with mean differences (between self-reported and measured values) ranging from $0.2 \mathrm{~cm}$ to $2.6 \mathrm{~cm}[18,45]$. Overall SDs of the mean differences ranged from 1.5 $\mathrm{cm}$ to $9.9 \mathrm{~cm}[40,62]$. Two studies found conflicting results [24,34]. In an Australian study, elderly people underestimated their height by a mean of $0.1 \mathrm{~cm}$ [34], while no difference between self-reported and measured height was found in a US study on health management program participants [24]. Furthermore, the overestimation of self-reported height seemed similar across the continents.

By sex. A total of 38 studies had data on men (Supplementary Table I). Height was overestimated in the majority of the studies, with mean differences ranging from $0.3 \mathrm{~cm}$ to $2.9 \mathrm{~cm}[22,70]$, while SDs of the mean differences ranged from $1.1 \mathrm{~cm}$ to $7.2 \mathrm{~cm}$ $[17,34]$. Two studies, however, found no differences between self-reported and measured height $[17,54]$, while in the Canadian Health Measures Survey conducted between 2007 and 2009, men underestimated their height by a mean of $1.2 \mathrm{~cm} \mathrm{[50].}$

Height was overestimated in the majority of the 50 studies that had data on women, with mean differences ranging from $0.2 \mathrm{~cm}$ to $4.0 \mathrm{~cm}$ (Supplementary Table I) $[20,55]$. SDs of the mean differences for women ranged from $0.9 \mathrm{~cm}$ to $7.7 \mathrm{~cm}[15,17]$. Height was underestimated in five studies, with mean differences ranging from $0.2 \mathrm{~cm}$ to $1.0 \mathrm{~cm}[17,34,64]$, while two studies found no difference $[18,47]$.

By BMI categories. A total of nine studies reported data on self-reported and measured height by BMI categories (Supplementary Table I). The majority of the studies showed that height was overestimated in all BMI categories (also in men and women). The overestimation, however, was increased towards the higher BMI categories. For example, in combined data from the NHANES surveys conducted between 2001 and 2006 ( $n=15,161)$, normal weight participants overestimated their height by a mean of $0.6 \mathrm{~cm}$, while obese participants overestimated their height by a mean of $1.2 \mathrm{~cm}[60]$.

\section{Weight}

Overall sample. A total of 60 studies reported data on self-reported and measured weight (Supplementary Table II). Of these, 25 studies had combined data on men and women. Weight was underestimated by selfreport in the majority of the studies, with mean differences ranging from $0.1 \mathrm{~kg}$ to $2.3 \mathrm{~kg}[11,16,63]$, while SDs of the mean differences ranged from 1.4 $\mathrm{kg}$ to $8.3 \mathrm{~kg}[32,69]$. Weight was overestimated by a mean of $0.1 \mathrm{~kg}$ in a Polish study with elderly participants [19]. Furthermore, underestimation of selfreported weight was lower in Asian studies and higher in North American studies when compared with studies conducted on the other continents.

By sex. Thirty-nine studies that reported data on men showed that weight was underestimated by selfreport. Mean differences ranged from $0.1 \mathrm{~kg}$ to 2.2 $\mathrm{kg}[16,50]$, while SDs of the mean differences ranged from $1.9 \mathrm{~kg}$ to $9.8 \mathrm{~kg}$ (Supplementary Table II) $[46,67]$. Four studies had conflicting results $[11,12,41,51]$. In the NHANES surveys (19761980, 1999-2000, 2001-2002, 2003-2004, and 2007-2008), men overestimated their weight by mean differences ranging from $0.1 \mathrm{~kg}$ to $0.4 \mathrm{~kg}$. In the NHANES conducted between 2005 and 2006, weight was underestimated by a mean of $0.1 \mathrm{~kg}$ $[11,12]$. Two Dutch studies that examined ethnic differences between self-reported and measured weight had inconsistent results. A study on Turkish, Moroccan, and Dutch people in the Netherlands found that weight was overestimated by Dutch $(0.1 \mathrm{~kg})$ and Turkish $(0.5 \mathrm{~kg})$ men but no difference was found in Moroccan men [41]. The other Dutch study found that weight was underestimated by Dutch $(1.2 \mathrm{~kg})$ men and men of African-Surinamese $(0.8 \mathrm{~kg})$ origin, while participants of Hindustani-Surinamese origin overestimated their weight by a mean of $0.3 \mathrm{~kg}$ [51].

Similarly, the majority of the 51 studies on women showed that weight was underestimated by selfreport with mean differences between self-reported and measured values ranging from $0.1 \mathrm{~kg}$ to $3.4 \mathrm{~kg}$ (Supplementary Table II) $[16,65]$. SDs of the mean differences for women ranged from $0.1 \mathrm{~kg}$ to $7.3 \mathrm{~kg}$ $[67,69]$. Women overestimated their weight in three studies, with mean differences ranging from $0.1 \mathrm{~kg}$ to $0.3 \mathrm{~kg}[46,54,71]$, while a study on Chinese nurses and a study on British engineering company workers found no difference between self-reported and measured weight $[29,47]$. In a Dutch study, women with Moroccan and Turkish origin underestimated their weight by a mean of $0.2 \mathrm{~kg}$ and by a mean of $0.4 \mathrm{~kg}$, respectively, whereas no difference was found among women of Dutch origin [41].

By BMI categories. A total of nine studies reported self-reported and measured weight by BMI categories (Supplementary Table II). In all of these studies, underestimation of weight increased towards the higher BMI categories and hence was highest among obese participants $[12,16,18,26,40,45,60,69,72]$. 
Table II. Mean differences in BMI (self-reported minus measured BMI).

\begin{tabular}{|c|c|c|c|}
\hline \multirow[t]{2}{*}{ Reference } & \multicolumn{3}{|c|}{ BMI $\left(\mathbf{k g} / \mathbf{m}^{2}\right)$} \\
\hline & total $(\mathrm{SD})$ & $\operatorname{men}(\mathrm{SD})$ & women (SD) \\
\hline \multicolumn{4}{|l|}{ Asia } \\
\hline \multicolumn{4}{|l|}{ Ikeda $[15]^{\mathrm{a}}$} \\
\hline All & - & $0.3(\mathrm{NC}) \downarrow$ & $0.7(\mathrm{NC}) \downarrow$ \\
\hline $\mathrm{BMI}<18.5$ & - & $0.7(1.7) \uparrow$ & $0.3(2.3) \uparrow$ \\
\hline BMI 18.5-24.9 & - & $0.2(\mathrm{NC}) \downarrow$ & $0.5(\mathrm{NC}) \downarrow$ \\
\hline BMI 25.0-27.4 & - & $0.9(2.6) \downarrow$ & $1.2(2.8) \downarrow$ \\
\hline BMI 27.5-29.9 & - & $1.1(1.3) \downarrow$ & $2.1(1.7) \downarrow$ \\
\hline $\mathrm{BMI} \geq 30$ & - & $1.6(2.1) \downarrow$ & $3.5(3.7) \downarrow$ \\
\hline \multicolumn{4}{|l|}{ Lu et al. [16] } \\
\hline All & $0.4(3.5) \downarrow$ & $0.4(1.2) \downarrow$ & $0.4(1.3) \downarrow$ \\
\hline $\mathrm{BMI}<18.5$ & $0.5(3.0) \uparrow$ & & \\
\hline BMI 18.5-24.9 & $0.1(2.4) \downarrow$ & & \\
\hline BMI 25.0-29.9 & $0.5(2.2) \downarrow$ & & \\
\hline $\mathrm{BMI} \geq 30$ & $1.2(2.7) \downarrow$ & & \\
\hline Anai et al. $[17]^{a}$ & - & $0.3(0.8) \downarrow$ & $0.2(1.5) \downarrow$ \\
\hline Xie et al. [29] & - & - & $0.1(1.4) \downarrow$ \\
\hline Yoon et al. [30] & - & $0.8(\mathrm{NR}) \downarrow$ & $1.6(\mathrm{NR}) \downarrow$ \\
\hline $\begin{array}{l}\text { Yong and Saito } \\
{[39]^{\mathrm{a}}}\end{array}$ & - & $0.7(1.2) \downarrow$ & $0.8(1.5) \downarrow$ \\
\hline \multicolumn{4}{|l|}{ Lee et al. [46] } \\
\hline All & - & $0.1(0.7) \downarrow$ & $0.1(0.5) \downarrow$ \\
\hline $\mathrm{BMI}<20.0$ & - & $0.5(0.8) \uparrow$ & $0.2(0.6) \uparrow$ \\
\hline BMI 20.0-24.9 & - & $0.0(0.7) \leftrightarrow$ & $0.0(0.6) \leftrightarrow$ \\
\hline BMI 25.0-29.9 & - & $0.2(0.8) \downarrow$ & $0.4(0.9) \downarrow$ \\
\hline $\mathrm{BMI} \geq 30.0$ & - & $0.8(1.5) \downarrow$ & $0.5(1.4) \downarrow$ \\
\hline \multicolumn{4}{|l|}{ Australia } \\
\hline \multicolumn{4}{|l|}{ Pursey et al. [26] } \\
\hline All & $0.6(0.1) \downarrow$ & $0.5(1.0) \downarrow$ & $0.6(1.0) \downarrow$ \\
\hline BMI 18.0-24.9 & $0.5(0.8) \downarrow$ & - & - \\
\hline $\mathrm{BMI} \geq 25$ & $0.9(1.2) \downarrow$ & - & - \\
\hline Pasalich et al. [34] & $0.4(1.6) \downarrow$ & $0.9(2.1) \downarrow$ & $0.1(1.2) \downarrow$ \\
\hline \multicolumn{4}{|c|}{ Yoong et al. $2013[35]$} \\
\hline informed $^{\mathrm{b}}$ & $0.7(1.9) \downarrow$ & - & - \\
\hline uninformed ${ }^{\mathrm{b}}$ & $0.6(2.2) \downarrow$ & - & - \\
\hline \multicolumn{4}{|l|}{ Hayes et al. [43] ${ }^{\mathrm{a}}$} \\
\hline $\begin{array}{l}\text { NHS, NNS } \\
1995\end{array}$ & - & $1.2(2.1) \downarrow$ & $1.4(2.0) \downarrow$ \\
\hline $\begin{array}{l}\text { NHS 2007- } \\
2008\end{array}$ & - & $0.6(2.1) \downarrow$ & $0.7(2.0) \downarrow$ \\
\hline \multicolumn{4}{|l|}{$\mathrm{Ng}$ et al. $[48]^{\mathrm{a}}$} \\
\hline All & $0.7(1.6) \downarrow$ & $0.9(3.4) \downarrow$ & $0.6(1.4) \downarrow$ \\
\hline $\mathrm{BMI}<20$ & - & $0.2(0.3) \downarrow$ & $0.2(1.2) \uparrow$ \\
\hline BMI 20.0-24.9 & - & $0.4(1.0) \downarrow$ & $0.2(0.9) \downarrow$ \\
\hline BMI 25.0-29.9 & - & $0.8(2.0) \downarrow$ & $0.6(1.3) \downarrow$ \\
\hline $\mathrm{BMI} \geq 30$ & - & $1.3(1.3) \downarrow$ & $1.1(1.6) \downarrow$ \\
\hline Burton et al. [52] & - & - & $0.1(1.6) \downarrow$ \\
\hline Taylor et al. [72] ${ }^{\mathrm{a}}$ & $1.0(1.7) \downarrow$ & $0.9(1.5) \downarrow$ & $1.1(1.9) \downarrow$ \\
\hline \multicolumn{4}{|c|}{ Europe } \\
\hline \multicolumn{4}{|c|}{ Celis-Morales et al. [18] } \\
\hline All & $0.3(\mathrm{NR}) \downarrow$ & $0.4(\mathrm{NR}) \downarrow$ & $0.2(\mathrm{NR}) \downarrow$ \\
\hline BMI 18.5-24.9 & $0.1(\mathrm{NR}) \downarrow$ & - & - \\
\hline BMI 25.0-29.9 & $0.5(\mathrm{NR}) \downarrow$ & - & - \\
\hline $\mathrm{BMI} \geq 30.0$ & $0.5(\mathrm{NR}) \downarrow$ & - & - \\
\hline $\begin{array}{l}\text { Niedzwiedzka } \\
\text { et al. [19] }\end{array}$ & $0.7(\mathrm{NR}) \downarrow$ & $0.6(\mathrm{NR}) \downarrow$ & $0.7(\mathrm{NR}) \downarrow$ \\
\hline Skeie et al. $[20]^{\mathrm{a}}$ & - & - & $0.6(1.5) \downarrow$ \\
\hline Wright et al. [21] & - & - & $0.7(1.2) \downarrow$ \\
\hline
\end{tabular}

Table II. (Continued)

\begin{tabular}{|c|c|c|c|}
\hline \multirow[t]{2}{*}{ Reference } & \multicolumn{3}{|c|}{ BMI $\left(\mathbf{k g} / \mathbf{m}^{2}\right)$} \\
\hline & total $(\mathrm{SD})$ & men $(S D)$ & women $(\mathrm{SD})$ \\
\hline \multicolumn{4}{|c|}{ Magnusson et al. [23] } \\
\hline $\begin{array}{l}\text { without } \\
\text { osteoarthritis }\end{array}$ & $0.8(\mathrm{NR}) \downarrow$ & - & - \\
\hline $\begin{array}{l}\text { with } \\
\text { osteoarthritis }\end{array}$ & $1.3(\mathrm{NR}) \downarrow$ & - & - \\
\hline $\begin{array}{l}\text { Tolonen et al. } \\
{[27]^{\text {a }}}\end{array}$ & - & $0.7(\mathrm{NR}) \downarrow$ & $0.8(\mathrm{NR}) \downarrow$ \\
\hline Lassale et al. [32] & $0.3(1.5) \downarrow$ & - & - \\
\hline \multicolumn{4}{|c|}{ Dijkshoorn et al. [41] $]^{\mathrm{a}}$} \\
\hline Dutch & - & $0.2(1.4) \downarrow$ & $0.2(1.1) \downarrow$ \\
\hline Turkish-Dutch & - & $0.4(1.5) \downarrow$ & $0.8(1.8) \downarrow$ \\
\hline $\begin{array}{l}\text { Moroccan- } \\
\text { Dutch }\end{array}$ & - & $0.2(1.6) \downarrow$ & $0.7(2.3) \downarrow$ \\
\hline $\begin{array}{l}\text { Großschädl et al. } \\
{[36]}\end{array}$ & $0.4(1.1) \downarrow$ & $0.4(1.1) \downarrow$ & $0.4(1.1) \downarrow$ \\
\hline \multicolumn{4}{|l|}{ Isidoro et al. [44] } \\
\hline All & - & - & $1.2(\mathrm{NR}) \downarrow$ \\
\hline $\mathrm{BMI}<25$ & - & - & $0.6(1.2) \downarrow$ \\
\hline BMI 25.0-29.9 & - & - & $1.2(1.3) \downarrow$ \\
\hline $\mathrm{BMI} \geq 30$ & - & - & $2.0(1.9) \downarrow$ \\
\hline Krul et al. $[45]^{\mathrm{a}}$ & & $0.6(1.2) \downarrow$ & $0.7(1.2) \downarrow$ \\
\hline Italy & - & $0.6(0.9) \downarrow$ & $1.1(1.0) \downarrow$ \\
\hline North America & - & $0.5(1.3) \downarrow$ & $0.8(1.1) \downarrow$ \\
\hline Netherlands & - & $0.7(1.2) \downarrow$ & $0.6(1.3) \downarrow$ \\
\hline Lois et al. [47] & $0.5(1.8) \downarrow$ & $0.5(1.8) \downarrow$ & $0.2(1.6) \downarrow$ \\
\hline Park et al. [49] $]^{a}$ & $0.9(\mathrm{NR}) \downarrow$ & $0.9(1.3) \downarrow$ & $0.9(1.4) \downarrow$ \\
\hline \multicolumn{4}{|l|}{$\begin{array}{l}\text { Van Valkengoed } \\
\text { et al. [51] }\end{array}$} \\
\hline Dutch & - & $0.7(1.3) \downarrow$ & $1.0(1.3) \downarrow$ \\
\hline $\begin{array}{l}\text { Hindustani- } \\
\text { Surinamese }\end{array}$ & - & $0.3(1.7) \downarrow$ & $1.0(1.5) \downarrow$ \\
\hline $\begin{array}{l}\text { African- } \\
\text { Surinamese }\end{array}$ & - & $0.6(1.7) \downarrow$ & $1.1(1.9) \downarrow$ \\
\hline Dahl et al. [53] & $0.6(1.3) \downarrow$ & - & - \\
\hline \multicolumn{4}{|c|}{ Oliveira et al. [59] $]^{\mathrm{a}}$} \\
\hline$\leq 45$ years & - & $0.7(1.4) \downarrow$ & $0.9(1.2) \downarrow$ \\
\hline$>45$ years & - & $0.9(1.3) \downarrow$ & $1.3(1.6) \downarrow$ \\
\hline $\begin{array}{l}\text { Danubio et al. } \\
\text { [61] }\end{array}$ & - & $1.1(1.2) \downarrow$ & $1.5(1.4) \downarrow$ \\
\hline \multicolumn{4}{|l|}{ Dekkers et al. [62] } \\
\hline All & $0.7(0.8) \downarrow$ & $0.7(0.8) \downarrow$ & $0.7(0.8) \downarrow$ \\
\hline BMI 25.0-29.9 & $0.6(0.7) \downarrow$ & - & - \\
\hline $\mathrm{BMI} \geq 30$ & $0.9(1.0) \downarrow$ & - & - \\
\hline Larsen et al. [65] & - & - & $1.5(1.0) \downarrow$ \\
\hline $\begin{array}{l}\text { Nyholm et al. } \\
{[70]^{\mathrm{a}}}\end{array}$ & - & $0.6(1.1) \downarrow$ & $0.8(1.1) \downarrow$ \\
\hline $\mathrm{BMI}<18.5$ & - & - & $0.4(\mathrm{NR}) \uparrow$ \\
\hline BMI 18.5-24.9 & - & $0.2(\mathrm{NR}) \downarrow$ & $0.5(\mathrm{NR}) \downarrow$ \\
\hline BMI 25.0-29.9 & - & $0.6(\mathrm{NR}) \downarrow$ & $0.9(\mathrm{NR}) \downarrow$ \\
\hline BMI 30.0-34.9 & - & $1.2(\mathrm{NR}) \downarrow$ & $1.2(\mathrm{NR}) \downarrow$ \\
\hline $\mathrm{BMI} \geq 35$ & - & $1.4(\mathrm{NR}) \downarrow$ & $1.5(\mathrm{NR}) \downarrow$ \\
\hline \multicolumn{4}{|l|}{ North America } \\
\hline Murphy et al. [24] & $0.4(1.7) \downarrow$ & & \\
\hline \multicolumn{4}{|l|}{ Poston et al. [25] } \\
\hline All & - & $0.1(0.9) \uparrow$ & - \\
\hline BMI 18.5-24.9 & - & $0.5(0.9) \uparrow$ & - \\
\hline BMI $25.0-29.9$ & - & $0.2(0.9) \uparrow$ & - \\
\hline BMI 30.0-34.9 & - & $0.1(0.9) \downarrow$ & - \\
\hline
\end{tabular}


Table II. (Continued)

\begin{tabular}{|c|c|c|c|}
\hline \multirow[t]{2}{*}{ Reference } & \multicolumn{3}{|l|}{ BMI $\left(\mathbf{k g} / \mathbf{m}^{2}\right)$} \\
\hline & total $(\mathrm{SD})$ & men $(\mathrm{SD})$ & women $(\mathrm{SD})$ \\
\hline $\mathrm{BMI} \geq 35$ & - & $0.9(0.9) \downarrow$ & - \\
\hline \multicolumn{4}{|c|}{ Hattori and Sturm [12] } \\
\hline \multicolumn{4}{|c|}{ NHANES 1999-2000 } \\
\hline All & $0.6(1.8) \downarrow$ & $0.3(1.5) \downarrow$ & $0.8(2.1) \downarrow$ \\
\hline $\mathrm{BMI}<30$ & $0.3(1.5) \downarrow$ & - & - \\
\hline $\mathrm{BMI} \geq 30$ & $1.3(2.4) \downarrow$ & - & - \\
\hline \multicolumn{4}{|c|}{ NHANES 2001-2002 } \\
\hline All & $0.5(2.0) \downarrow$ & $0.2(2.0) \downarrow$ & $0.8(1.9) \downarrow$ \\
\hline $\mathrm{BMI}<30$ & $0.2(1.7) \downarrow$ & - & - \\
\hline $\mathrm{BMI} \geq 30$ & $1.2(2.4) \downarrow$ & - & - \\
\hline \multicolumn{4}{|c|}{ NHANES 2003-2004 } \\
\hline All & $0.5(1.7) \downarrow$ & $0.3(1.5) \downarrow$ & $0.6(1.8) \downarrow$ \\
\hline $\mathrm{BMI}<30$ & $0.2(1.3) \downarrow$ & - & - \\
\hline $\mathrm{BMI} \geq 30$ & $1.1(2.2) \downarrow$ & - & - \\
\hline \multicolumn{4}{|c|}{ NHANES 2005-2006 } \\
\hline All & $0.6(1.8) \downarrow$ & $0.4(1.7) \downarrow$ & $0.7(1.8) \downarrow$ \\
\hline $\mathrm{BMI}<30$ & $0.2(1.3) \downarrow$ & - & - \\
\hline $\mathrm{BMI} \geq 30$ & $1.2(2.3) \downarrow$ & - & - \\
\hline \multicolumn{4}{|c|}{ NHANES 2007-2008 } \\
\hline All & $0.6(1.7) \downarrow$ & $0.4(1.6) \downarrow$ & $0.8(1.8) \downarrow$ \\
\hline $\mathrm{BMI}<30$ & $0.2(1.3) \downarrow$ & - & - \\
\hline $\mathrm{BMI} \geq 30$ & $1.3(2.2) \downarrow$ & - & - \\
\hline \multicolumn{4}{|c|}{ Stommel and Osier $[14]^{\mathrm{a}}$} \\
\hline \multicolumn{4}{|c|}{ NHANES $1988-1994$} \\
\hline All & $0.6(3.3) \downarrow$ & - & - \\
\hline BMI $18.5-24.9$ & $0.1(\mathrm{NR}) \downarrow$ & - & - \\
\hline BMI 25.0-29.9 & $0.6(\mathrm{NR}) \downarrow$ & - & - \\
\hline BMI 29.9-34.9 & $1.3(\mathrm{NR}) \downarrow$ & - & - \\
\hline BMI 35.0-39.9 & $1.8(\mathrm{NR}) \downarrow$ & - & - \\
\hline $\mathrm{BMI} \geq 40$ & $3.5(\mathrm{NR}) \downarrow$ & - & - \\
\hline \multicolumn{4}{|c|}{ NHANES 2005-2008 } \\
\hline All & $0.6(2.7) \downarrow$ & - & - \\
\hline BMI 18.5-24.9 & $0.0(\mathrm{NR}) \leftrightarrow$ & - & - \\
\hline BMI $25.0-29.9$ & $0.6(\mathrm{NR}) \downarrow$ & - & - \\
\hline BMI 29.9-34.9 & $1.0(\mathrm{NR}) \downarrow$ & - & - \\
\hline BMI 35.0-39.9 & $1.6(\mathrm{NR}) \downarrow$ & - & - \\
\hline $\mathrm{BMI} \geq 40$ & $2.3(\mathrm{NR}) \downarrow$ & - & - \\
\hline \multicolumn{4}{|l|}{ Lin et al. [37] ${ }^{\mathrm{a}}$} \\
\hline CATI ${ }^{c}$ & - & - & $0.7(0.8) \downarrow$ \\
\hline questionnaire $^{c}$ & - & - & $(\mathrm{NR})$ \\
\hline $\begin{array}{l}\text { Wen and } \\
\text { Kowaleski-Jones } \\
{[38]}\end{array}$ & - & $0.4(\mathrm{NR}) \downarrow$ & $0.8(\mathrm{NR}) \downarrow$ \\
\hline \multicolumn{4}{|c|}{ Bes-Rastrollo et al. [40] } \\
\hline All & $0.6(2.2) \downarrow$ & $0.5(1.8) \downarrow$ & $0.7(2.5) \downarrow$ \\
\hline $\mathrm{BMI}<30$ & $0.4(2.0) \downarrow$ & - & - \\
\hline $\mathrm{BMI} \geq 30$ & $0.9(2.8) \downarrow$ & - & - \\
\hline \multicolumn{4}{|c|}{ Griebeler et al. [42] } \\
\hline All & - & - & $1.3(1.6) \downarrow$ \\
\hline $\mathrm{BMI}<20$ & - & - & $0.2(0.8) \downarrow$ \\
\hline BMI 20.0-24.9 & - & - & $0.8(1.4) \downarrow$ \\
\hline BMI 25.0-29.9 & - & - & $1.4(1.5) \downarrow$ \\
\hline $\mathrm{BMI}>30$ & - & - & $2.4(1.6) \downarrow$ \\
\hline \multicolumn{4}{|l|}{ Shields et al. [50] a } \\
\hline CCHS 2005 & - & $1.0(\mathrm{NR}) \downarrow$ & $1.3(\mathrm{NR}) \downarrow$ \\
\hline CCHS 2008 & - & $0.6(\mathrm{NR}) \downarrow$ & $0.9(\mathrm{NR}) \downarrow$ \\
\hline $\begin{array}{l}\text { CHMS } \\
2007-2009\end{array}$ & - & $1.2(\mathrm{NR}) \downarrow$ & $1.5(\mathrm{NR}) \downarrow$ \\
\hline
\end{tabular}

Table II. (Continued)

\begin{tabular}{|c|c|c|c|}
\hline \multirow[t]{2}{*}{ Reference } & \multicolumn{3}{|l|}{ BMI $\left(\mathbf{k g} / \mathbf{m}^{2}\right)$} \\
\hline & total $(\mathrm{SD})$ & men $(\mathrm{SD})$ & women $(\mathrm{SD})$ \\
\hline \multicolumn{4}{|c|}{ Connor Gorber and Tremblay $[11]^{\mathrm{a}}$} \\
\hline $\begin{array}{l}\text { NHANES } \\
1976-1980\end{array}$ & $0.4(\mathrm{NR}) \downarrow$ & $0.3(\mathrm{NR}) \downarrow$ & $0.6(\mathrm{NR}) \downarrow$ \\
\hline $\begin{array}{l}\text { NHANES } \\
1988-1994\end{array}$ & $0.5(\mathrm{NR}) \downarrow$ & $0.3(\mathrm{NR}) \downarrow$ & $0.7(\mathrm{NR}) \downarrow$ \\
\hline $\begin{array}{l}\text { NHANES } \\
2003-2004\end{array}$ & $0.5(\mathrm{NR}) \downarrow$ & $0.3(\mathrm{NR}) \downarrow$ & $0.7(\mathrm{NR}) \downarrow$ \\
\hline $\begin{array}{l}\text { CHHS } \\
1986-1992\end{array}$ & $0.8(\mathrm{NR}) \downarrow$ & $0.7(\mathrm{NR}) \downarrow$ & $0.9(\mathrm{NR}) \downarrow$ \\
\hline CCHS 2005 & $1.1(\mathrm{NR}) \downarrow$ & $1.0(\mathrm{NR}) \downarrow$ & $1.2(\mathrm{NR}) \downarrow$ \\
\hline $\begin{array}{l}\text { Fillenbaum et al. } \\
\text { [54] }\end{array}$ & - & $0.7(1.8) \downarrow$ & $0.9(2.0) \downarrow$ \\
\hline Meng et al. [56] ${ }^{\mathrm{a}}$ & $1.2(8.2) \downarrow$ & - & - \\
\hline $\begin{array}{l}\text { Ahluwalia et al. } \\
\text { [57] }\end{array}$ & - & - & $0.8(\mathrm{NR}) \downarrow$ \\
\hline \multicolumn{4}{|c|}{ Merrill and Richardson [58] $]^{\mathrm{a}}$} \\
\hline All & - & $0.3(1.8) \downarrow$ & $0.8(1.9) \downarrow$ \\
\hline $\mathrm{BMI}<18.5$ & - & $1.0(2.0) \uparrow$ & $0.3(1.1) \uparrow$ \\
\hline BMI 18.5-24.9 & - & $0.3(2.4) \uparrow$ & $0.2(1.5) \downarrow$ \\
\hline BMI 25.0-29.9 & - & $0.4(1.7) \downarrow$ & $0.9(2.3) \downarrow$ \\
\hline $\mathrm{BMI} \geq 30$ & - & $1.1(2.6) \downarrow$ & $1.6(3.3) \downarrow$ \\
\hline \multicolumn{4}{|l|}{$\begin{array}{l}\text { Stommel and } \\
\text { Schoenborn [60] }\end{array}$} \\
\hline All & $0.6(2.5) \downarrow$ & - & - \\
\hline $\mathrm{BMI}<18.5$ & $0.6(\mathrm{NR}) \uparrow$ & - & - \\
\hline BMI 18.5-24.9 & $0.0(\mathrm{NR}) \leftrightarrow$ & - & - \\
\hline BMI 25.0-29.9 & $0.5(\mathrm{NR}) \downarrow$ & - & - \\
\hline BMI 30.0-39.9 & $1.2(\mathrm{NR}) \downarrow$ & - & - \\
\hline $\mathrm{BMI} \geq 40$ & $2.1(\mathrm{NR}) \downarrow$ & - & - \\
\hline $\begin{array}{l}\text { Elgar and Stewart } \\
\text { [63 }^{\text {a }}\end{array}$ & $1.2(4.2) \downarrow$ & $1.0(4.0) \downarrow$ & $1.3(4.2) \downarrow$ \\
\hline Jeffery et al. [64] & - & - & - \\
\hline Paradis et al. [66] & - & $0.4(1.4) \downarrow$ & $0.7(1.4) \downarrow$ \\
\hline \multicolumn{4}{|c|}{ Sahyoun et al. [67] ${ }^{a}$} \\
\hline $\mathrm{BMI}<18.5$ & - & $0.8(3.3) \uparrow$ & $0.4(2.4) \uparrow$ \\
\hline BMI 18.5-24.9 & - & $1.0(2.8) \downarrow$ & $0.6(2.8) \downarrow$ \\
\hline BMI 25-29.9 & - & $0.8(3.2) \downarrow$ & $1.1(2.9) \downarrow$ \\
\hline $\mathrm{BMI} \geq 30$ & - & $1.5(2.1) \downarrow$ & $1.9(2.5) \downarrow$ \\
\hline \multicolumn{4}{|l|}{ Shields et al. [5] ${ }^{\mathrm{a}}$} \\
\hline All & $1.1(1.7) \downarrow$ & $0.9(2.3) \downarrow$ & $1.2(2.5) \downarrow$ \\
\hline $\mathrm{BMI}<18.5$ & - & $2.6(4.9) \uparrow$ & $0.2(1.6) \uparrow$ \\
\hline BMI 18.5-24.9 & - & $0.1(2.1) \downarrow$ & $0.5(1.7) \downarrow$ \\
\hline BMI 25.0-29.9 & - & $1.0(2.2) \downarrow$ & $1.4(2.7) \downarrow$ \\
\hline BMI 30.0-34.9 & - & $1.9(3.0) \downarrow$ & $2.1(3.8) \downarrow$ \\
\hline BMI 35.0-39.9 & - & $2.7(2.6) \downarrow$ & $3.0(5.0) \downarrow$ \\
\hline $\mathrm{BMI} \geq 40$ & - & $4.0(3.2) \downarrow$ & $5.0(7.5) \downarrow$ \\
\hline $\begin{array}{l}\text { Brunner Huber } \\
\text { [68] }\end{array}$ & - & - & $0.8(1.9) \downarrow$ \\
\hline \multicolumn{4}{|l|}{$\begin{array}{l}\text { McAdams et al. } \\
\text { [69] }\end{array}$} \\
\hline All & $0.5(3.1) \downarrow$ & $0.2(2.9) \downarrow$ & $0.7(2.9) \downarrow$ \\
\hline $\mathrm{BMI}<25$ & $0.0(\mathrm{NR}) \leftrightarrow$ & $0.3(\mathrm{NR}) \uparrow$ & $0.2(\mathrm{NR}) \downarrow$ \\
\hline BMI 25.0-29.9 & $0.6(\mathrm{NR}) \downarrow$ & $0.4(\mathrm{NR}) \downarrow$ & $0.9(\mathrm{NR}) \downarrow$ \\
\hline $\mathrm{BMI} \geq 30$ & $1.8(\mathrm{NR}) \downarrow$ & $1.4(\mathrm{NR}) \downarrow$ & $2.1(\mathrm{NR}) \downarrow$ \\
\hline \multicolumn{4}{|l|}{ South America } \\
\hline $\begin{array}{l}\text { Lucca and Moura } \\
\text { [55] }^{\mathrm{a}}\end{array}$ & - & $0.4(\mathrm{NR}) \downarrow$ & $1.5(\mathrm{NR}) \downarrow$ \\
\hline $\mathrm{BMI} \leq 24.9$ & - & $0.6(\mathrm{NR}) \uparrow$ & $0.9(\mathrm{NR}) \downarrow$ \\
\hline
\end{tabular}


Table II. (Continued)

\begin{tabular}{llll}
\hline Reference & \multicolumn{3}{l}{ BMI $\left(\mathbf{k g} / \mathbf{m}^{2}\right)$} \\
\cline { 2 - 4 } & total $(\mathrm{SD})$ & men $(\mathrm{SD})$ & women (SD) \\
\hline BMI 25.0-29.9 & - & $0.7(\mathrm{NR}) \downarrow$ & $1.3(\mathrm{NR}) \downarrow$ \\
BMI $\geq 30$ & - & $1.9(\mathrm{NR}) \downarrow$ & $2.4(\mathrm{NR}) \downarrow$ \\
\hline
\end{tabular}

$\uparrow$, overestimated by self-report; $\downarrow$, underestimated by self-report; BMI, body mass index; CATI, computer-assisted telephone interview; CCHS, Canadian Community Health Survey; CHMS, Canadian Health Measures Survey; NC, not calculated(standard deviation could not be calculated); NHANES, National Health and Nutrition Examination Survey; NHS, National Health Survey; NNS, National Nutrition Survey; NR, not reported; SD, standard deviation.

${ }^{\mathrm{a}}$ For a general population.

bParticipants were notified before (informed) or after (uninformed) self-reporting that they would be measured.

'Self-reporting was based on CATI or a self-administered questionnaire.

This tendency was also seen for men and women. For example, in the Canadian Community Health Survey data $(n=4537)$, women of normal weight underestimated their weight by a mean of $1 \mathrm{~kg}$, while obese $(\mathrm{BMI} \geq 40)$ women underestimated their weight by a mean of $9 \mathrm{~kg}$ [5]. Men of normal weight men underestimated their weight by a mean of $0.3 \mathrm{~kg}$ and obese men by a mean of $5 \mathrm{~kg}$. Furthermore, in many studies underweight participants (particularly men) overestimated their weight $[5,15,58,60,67,70,72]$.

\section{Body mass index}

Overall sample. Data on self-reported (calculated from self-reported height and weight) and measured (calculated from measured height and weight) BMI was reported in 57 studies (Table II). Of these, 29 studies had data on the overall sample; in all of these studies BMI was underestimated by self-report by mean differences between self-reported and measured values ranging from $0.3 \mathrm{~kg} / \mathrm{m}^{2}$ to $1.2 \mathrm{~kg} / \mathrm{m}^{2}$ $[18,32,56,63]$. The overall SDs of the mean differences between self-reported and measured values ranged from $0.1 \mathrm{~kg} / \mathrm{m}^{2}$ to $8.2 \mathrm{~kg} / \mathrm{m}^{2}$ [26,56]. Furthermore, underestimation of self-reported BMI seemed to be lower in Asian studies and higher in North American studies when compared to the other continents.

By sex. A total of 37 studies reported data on men. BMI was underestimated in the majority of the studies with mean differences between self-reported and measured values ranging from $0.1 \mathrm{~kg} / \mathrm{m}^{2}$ to $1.2 \mathrm{~kg} / \mathrm{m}^{2}$ (Table II) $[43,46,50]$. SDs of the mean differences for men ranged from $0.7 \mathrm{~kg} / \mathrm{m}^{2}$ to $4.0 \mathrm{~kg} / \mathrm{m}^{2}[46,63]$. However, among US firefighters BMI derived from self-reported measures was overestimated by a mean of $0.1 \mathrm{~kg} / \mathrm{m}^{2}$ [25].

All 46 studies that reported data on women showed that self-reported BMI was underestimated with mean differences between self-reported and measured values ranging from $0.1 \mathrm{~kg} / \mathrm{m}^{2}$ to $1.6 \mathrm{~kg} / \mathrm{m}^{2}$ $[29,30,34,46,52]$. SDs of the mean differences for women ranged from $0.5 \mathrm{~kg} / \mathrm{m}^{2}$ to $4.2 \mathrm{~kg} / \mathrm{m}^{2}$ [46,63].

By BMI categories. A total of 20 studies reported data on the mean differences between self-reported and measured BMI by BMI categories (Table II). Self-reported BMI was overestimated by underweight participants, whereas underestimation of BMI increased towards the higher BMI categories (particularly in women). For example, in the Canadian Health Community Survey $(n=4537)$, self-reported BMI was underestimated by a mean of $0.5 \mathrm{~kg} / \mathrm{m}^{2}$ in normal weight women, while women with a BMI over 40 underestimated their BMI by a mean of 5.0 $\mathrm{kg} / \mathrm{m}^{2}$ [5]. As for men, normal weight men underestimated their BMI by a mean of $0.1 \mathrm{~kg} / \mathrm{m}^{2}$, while men with a BMI over 40 underestimated their BMI by a mean of $4.0 \mathrm{~kg} / \mathrm{m}^{2}$.

\section{Differences in prevalence of overweight and} obesity

Prevalence of overweight in the overall sample. The mean differences in the prevalence of overweight and obesity, calculated from BMI based on self-reported values and measured values were derived from 34 studies (Table III). Of these, 12 studies had data on the overall sample. The majority of the studies showed that the prevalence of overweight was underestimated by self-report ranging from $1.8 \%$-points to $9.3 \%$-points [5,63]. In four studies, the prevalence of overweight was overestimated (mean differences ranged from $0.2 \%$-points to $5.1 \%$-points) $[23,36,48,62]$. An Australian study on general practitioners' appointment attendees found no difference in the prevalence of overweight [35]. Furthermore, the underestimation of overweight prevalence seemed to be lower in Australian studies than in the studies conducted on the other continents.

Prevalence of overweight according to sex. A total of 19 studies reported data on men (Table III). With the exception of two studies $[36,62]$, the prevalence of overweight was underestimated when based on selfreported BMI, ranging from $0.5 \%$-points to $9.4 \%$-points $[5,48]$. The prevalence of overweight was overestimated by $2.0 \%$-points among Austrian health check attendees and by $4.1 \%$-points among Dutch overweight working population $[36,62]$. 
Table III. Mean differences in the prevalence of overweight and obesity.

\begin{tabular}{|c|c|c|c|c|c|c|}
\hline \multirow[t]{3}{*}{ Reference } & \multicolumn{6}{|c|}{ Mean difference in prevalence, $\%$} \\
\hline & \multicolumn{3}{|c|}{ overweight (BMI 25.0-29.9 kg/m²) } & \multicolumn{3}{|c|}{ obese $\left(\mathrm{BMI} \geq 30.0 \mathrm{~kg} / \mathrm{m}^{2}\right)$} \\
\hline & total & men & women & total & men & women \\
\hline \multicolumn{7}{|l|}{ Asia } \\
\hline Ikeda $[15]^{\mathrm{a}}$ & - & $3.7 \downarrow$ & $5.1 \downarrow$ & - & $0.3 \downarrow$ & $1.3 \downarrow$ \\
\hline Lu et al. [16] & $5.8 \downarrow$ & $4.3 \downarrow$ & $7.8 \downarrow$ & $1.3 \downarrow$ & $0.6 \downarrow$ & $2.1 \downarrow$ \\
\hline Xie et al. [29] & - & - & $1.4 \downarrow^{\mathrm{b}}$ & - & - & $0.7 \downarrow b$ \\
\hline Yoon et al. [30] & - & $19.5 \downarrow c$ & $17.9 \downarrow^{c}$ & - & - & - \\
\hline Yong and Saito [39] ${ }^{\mathrm{a}}$ & - & $6.2 \downarrow$ & $4.9 \downarrow$ & - & $0.7 \downarrow$ & $2.9 \downarrow$ \\
\hline Lee et al. [46] & & $1.1 \downarrow c$ & $2.0 \downarrow_{c}$ & & & \\
\hline \multicolumn{7}{|l|}{ Australia } \\
\hline Yoong et al. 2013 [35] & 0 & - & - & $5.0 \downarrow$ & - & - \\
\hline Hayes et al. $[43]^{\mathrm{a}}$ & - & $1.4 \downarrow$ & $3.3 \downarrow$ & - & $3.9 \downarrow$ & $3.4 \downarrow$ \\
\hline $\mathrm{Ng}$ et al. $[48]^{\mathrm{a}}$ & $0.8 \uparrow$ & $0.5 \downarrow$ & $2.2 \uparrow$ & $5.6 \downarrow$ & $6.8 \downarrow$ & $4.4 \downarrow$ \\
\hline Burton et al. [52] & - & - & $4.4 \downarrow$ & - & - & $0.6 \downarrow$ \\
\hline Taylor et al. $[72]^{\mathrm{a}}$ & $2.1 \downarrow$ & $0.7 \downarrow$ & $3.7 \downarrow$ & $7.2 \downarrow$ & $7.6 \downarrow$ & $6.9 \downarrow$ \\
\hline \multicolumn{7}{|l|}{ Europe } \\
\hline Celis-Morales et al. [18] & $2.8 \downarrow$ & - & - & $0.7 \downarrow$ & - & - \\
\hline Niedzwiedzka et al. [19] ${ }^{a}$ & $6.0 \downarrow$ & $6.0 \downarrow$ & $6.0 \downarrow$ & $5.0 \downarrow$ & $8.0 \downarrow$ & $2.0 \downarrow$ \\
\hline Skeie et al. $[20]^{a}$ & - & - & $8.2 \downarrow$ & - & - & $1.9 \downarrow$ \\
\hline \multicolumn{7}{|l|}{ Magnusson et al. [23] } \\
\hline without osteoarthritis & $3.3 \uparrow$ & - & - & $10.6 \downarrow$ & - & - \\
\hline with osteoarthritis & $5.1 \uparrow$ & - & - & $13.4 \downarrow$ & - & - \\
\hline Tolonen et al. [27] ${ }^{\mathrm{a}}$ & - & $4.4 \downarrow$ & $6.1 \downarrow$ & - & $4.4 \downarrow$ & $4.2 \downarrow$ \\
\hline Lassale et al. [32] & $2.2 \downarrow$ & - & - & $1.7 \downarrow$ & - & - \\
\hline May et al. $[33]^{\mathrm{a}}$ & - & $1.0 \downarrow$ & $0.6 \downarrow$ & - & $0.6 \downarrow$ & $0.7 \downarrow$ \\
\hline \multicolumn{7}{|l|}{ Dijkshoorn et al. [41] ${ }^{\mathrm{a}}$} \\
\hline Dutch & - & - & - & - & $2.9 \downarrow$ & $2.5 \downarrow$ \\
\hline Turkish-Dutch & - & - & - & - & $3.4 \downarrow$ & $5.5 \downarrow$ \\
\hline Moroccan-Dutch & - & - & - & - & $4.2 \downarrow$ & $5.3 \downarrow$ \\
\hline Großschädl et al. [36] & $0.2 \uparrow$ & $2.0 \uparrow$ & $1.8 \downarrow$ & $2.9 \downarrow$ & $2.8 \downarrow$ & $3.3 \downarrow$ \\
\hline Isidoro et al. [44] & - & - & $3.0 \downarrow$ & - & - & $8.4 \downarrow$ \\
\hline \multicolumn{7}{|l|}{ Krul et al. $[45]^{\mathrm{a}}$} \\
\hline Italy & - & $7.1 \downarrow$ & $3.6 \downarrow$ & - & $2.2 \downarrow$ & $2.4 \downarrow$ \\
\hline North America & - & $3.4 \downarrow$ & $2.8 \downarrow$ & - & $2.7 \downarrow$ & $2.7 \downarrow$ \\
\hline Netherlands & - & $1.5 \downarrow$ & $2.9 \downarrow$ & - & $3.7 \downarrow$ & $1.5 \downarrow$ \\
\hline Park et al. [49] ${ }^{\mathrm{a}}$ & $4.6 \downarrow$ & - & - & $6.8 \downarrow$ & - & - \\
\hline Danubio et al. [61] & - & $8.1 \downarrow$ & $7.8 \downarrow$ & - & $3.3 \downarrow$ & $0.9 \downarrow$ \\
\hline Dekkers et al. [62] & $3.4 \uparrow$ & $4.1 \uparrow$ & $1.8 \uparrow$ & $6.9 \downarrow$ & $6.8 \downarrow$ & $6.9 \downarrow$ \\
\hline Larsen et al. [65] & - & - & - & - & - & $3.4 \downarrow$ \\
\hline Nyholm et al. $[70]^{\mathrm{a}}$ & - & - & - & - & $5.3 \downarrow$ & $4.9 \downarrow$ \\
\hline \multicolumn{7}{|l|}{ North America } \\
\hline Griebeler et al. [42] & - & - & $2.6 \downarrow$ & - & - & $11.9 \downarrow$ \\
\hline Fillenbaum et al. [54] & - & - & - & - & - & - \\
\hline African-American & $2.5 \downarrow$ & - & - & $5.4 \downarrow$ & - & - \\
\hline White & $3.0 \downarrow$ & & & $6.5 \downarrow$ & & \\
\hline Ahluwalia et al. [57] & - & - & $2.2 \downarrow$ & - & - & - \\
\hline Elgar and Stewart [63] & $1.8 \downarrow$ & $0.6 \downarrow$ & $3.0 \downarrow$ & $7.6 \downarrow$ & $8.8 \downarrow$ & $6.4 \downarrow$ \\
\hline Shields et al. [5] $]^{\mathrm{a}}$ & $9.3 \downarrow$ & $9.4 \downarrow$ & $9.1 \downarrow$ & $7.4 \downarrow$ & $8.8 \downarrow$ & $6.0 \downarrow$ \\
\hline Brunner Huber [68] & - & - & $0.4 \uparrow$ & - & - & $2.4 \uparrow$ \\
\hline \multicolumn{7}{|l|}{ South America } \\
\hline Tsai et al. [28] & - & - & $11.4 \downarrow^{c}$ & - & - & $4.6 \downarrow$ \\
\hline Lucca and Moura [55] & - & - & - & - & $2.5 \downarrow$ & $6.4 \downarrow$ \\
\hline
\end{tabular}

$\uparrow$, overestimated by self-report; $\downarrow$, underestimated by self-report. ${ }^{\mathrm{a}}$ For a general population.

bBMI for overweight $23-25 \mathrm{~kg} / \mathrm{m}^{2}$, BMI for obese $\geq 25 \mathrm{~kg} / \mathrm{m}^{2}$.

Included both overweight and obese participants. 
In the majority of the 21 studies that reported data on women, the prevalence of overweight was underestimated by self-report, ranging from $0.6 \%$-points to $11.4 \%$-points (Table III) $[28,33]$. In three studies, the prevalence of overweight was overestimated by $0.4 \%$-points [68], $1.8 \%$-points [62], and by $2.2 \%$-points [48].

Prevalence of obesity in overall sample. The prevalence of obesity was underestimated by self-report in all 13 studies with data on the overall sample, ranging from $0.7 \%$-points to $13.4 \%$-points (Table III) $[18,23]$. Furthermore, underestimation of obesity prevalence was lower in Asian studies and higher in North American studies than those in the other continents.

Prevalence of obesity according to sex. A total of 18 studies reported data on men and all studies showed that the prevalence of obesity was underestimated by self-report, ranging from $0.3 \%$-points to $8.8 \%$-points (Table III) [5,15,63]. Furthermore, in a South Korean study that included participants from middle age onwards, the prevalence of obesity (defined as BMI $\geq 25.0 \mathrm{~kg} / \mathrm{m}^{2}$ ) was underestimated in men by $20 \%$-points and in women by $18 \%$-points [30].

In the 26 studies that reported data on women, with the exception of one study the prevalence of obesity was underestimated when based on selfreported BMI [68], ranging from $0.6 \%$-points to $11.9 \%$-points (Table III) $[42,52]$. In a US study on women using birth control, the self-reported prevalence of obesity was overestimated by $2.4 \%$-points [68].

\section{Discussion}

This literature review of studies published between 2006 and 2017 revealed a tendency for underestimation of self-reported weight and overestimation of height compared with the measured values. These biases were evident for both sexes. Furthermore, BMI derived from self-reported height and weight was underestimated and consequently, a clear tendency for underestimation of the prevalence of overweight and obesity was found. The bias tended to be greater for those in the higher BMI groups. Furthermore, continent differences on self-reported bias were also found.

The bias observed here was similar to those of the systematic review by Connor Gorber et al. [6], which compared self-reported and measured height, weight and BMI based on 64 studies on men and women published between 1982 and 2005. The studies included in our review, however, showed a slightly narrower range for the overall mean differences for weight $(0.1-2.3 \mathrm{~kg}$ vs $0.1-3.5 \mathrm{~kg})$, height $(0.1-2.6$ $\mathrm{cm}$ vs $0.6-7.5 \mathrm{~cm})$ and $\mathrm{BMI}\left(0.3-1.2 \mathrm{~kg} / \mathrm{m}^{2}\right.$ vs $0.2-$ $\left.1.8 \mathrm{~kg} / \mathrm{m}^{2}\right)$. Similar tendencies were also seen for men and women separately.

Few recent studies have examined the temporal change in the bias of self-reported BMI [11-14]. The Surveys of Lifestyle Attitudes and Nutrition (SLÁN) conducted in Ireland between 1998 and 2007 found that the bias in self-reported BMI increased over time [13]. The self-reported BMI and obesity bias stayed relatively constant in the US between 1976 and 2004 [11]. In Canada, however, the bias increased over the time period between 1986 and 2005 [11]. Analysis of the NHAHES survey data from 1999 to 2008 did not reveal a temporal change in self-reported bias [12]. Furthermore, another US study compared the NHANES surveys conducted between 1988 and 1994 with surveys conducted between 2005 and 2008 . This study revealed that the bias in self-reported BMI had increased slightly over this time period [14]. However, among obese individuals, self-reporting bias in BMI had declined, though the bias was still highest among obese individuals when compared with normal and underweight individuals.

Furthermore, our review indicated that underestimation of self-reported weight was lower and overestimation of height was higher among Asian studies than those from studies conducted on the other continents. Asian studies also showed a lower underestimation of BMI by self-report than studies from the other continents. This may be due to cultural differences. Asian people tend to be shorter than people from western; obesity is also not as common in Asia as it in western countries. Most of the previous studies were conducted in Europe and in North America, while only a few studies were conducted in Asia, Australia, and Africa.

During the past decade, a number of studies have been published on the bias of self-reported anthropometrics, which reflects the significance of this matter. The authors of the systematic review [6] emphasized the need for common criteria for data analysis and for reporting anthropometric results from studies. However, some of the studies included in the present review lacked vital information that may have had an effect on the results, such as elapsed time between measurements or order of the measurements. Selfreported information should always be collected before objective measurements are conducted. Uniformity concerning the study procedures facilitate comparison of the studies.

Furthermore, it should be noted that because the literature search for this review was conducted solely on PubMed (as this is the largest available data 
resource) and because this review was not a systematic review, there is a possibility that we may have missed some relevant publications.

In conclusion, consistent with previous literature this review showed a tendency for underestimation of self-reported BMI and consequently the prevalence of overweight and obesity. The self-reporting bias was higher among overweight and obese individuals than among underweight or those of normal weight. Overweight and obese individuals are a high risk group with regards to NCDs. Thus, with globally growing obesity rates it is of utmost importance to obtain accurate information on the prevalence of overweight and obesity to assess whether the global targets for NCD prevention and for stopping obesity increases will be reached. Therefore, measured anthropometrics may provide a more reliable tool for assessing the prevalence of obesity.

\section{Acknowledgements}

The authors are grateful to the information specialist Mari Mäkinen for her help with planning the database search and for Derek Ho for polishing the English.

\section{Declaration of conflicting interests}

The author(s) declared no potential conflicts of interest with respect to the research, authorship, and/ or publication of this article.

\section{Funding}

The authors disclosed receipt of the following financial support for the research, authorship, and/or publication of this article: This work was supported by the European Commission, DG SANTÉ through BRIDGE Health project (grant number 664692), the Finnish Cultural Foundation, and The Juho Vainio Foundation.

\section{References}

[1] World Health Organization. Obesity:preventing and managing the global epidemic. Report of a WHO consultation. Geneva, Switzerland: World Health Organization, 2000.

[2] Seidell JC and Halberstadt J. The global burden of obesity and the challenges of prevention. Ann Nutr Metab 2015;66(Suppl. 2):7-12.

[3] World Health Organization. Global action plan for the prevention and control of noncommunicable diseases 2013-2020. Geneva, Switzerland: World Health Organization, 2013.

[4] Chiolero A, Peytremann-Bridevaux I and Paccaud F. Associations between obesity and health conditions may be overestimated if self-reported body mass index is used. Obes Rev 2007;8:373-4.

[5] Shields M, Connor Gorber S and Tremblay MS. Estimates of obesity based on self-report versus direct measures. Health Rep 2008, 19, 61-79

[6] Connor Gorber S, Tremblay M, Moher D, et al. A comparison of direct vs. self-report measures for assessing height, weight and body mass index: a systematic review. Obes Rev 2007, 8, 307-326.

[7] Larson MR. Social desirability and self-reported weight and height. Int 7 Obes Relat Metab Disord 2000, 24, 663-665.

[8] Madrigal H, Sanchez-Villegas A, Martinez-Gonzalez MA, et al. Underestimation of body mass index through perceived body image as compared to self-reported body mass index in the European Union. Public Health 2000, 114, 468473.

[9] Shoham DA, Hammond R, Rahmandad H, et al. Modeling social norms and social influence in obesity. Curr Epidemiol Rep 2015, 2, 1-79.

[10] Robinson E and Christiansen P. The changing face of obesity: exposure to and acceptance of obesity. Obesity 2014, 22, 1380-1386.

[11] Connor Gorber S and Tremblay MS. The bias in selfreported obesity from 1976 to 2005 : a Canada-US comparison. Obesity 2010, 18, 354-361.

[12] Hattori A and Sturm R. The obesity epidemic and changes in self-report biases in BMI. Obesity 2013, 21, 856-860.

[13] Shiely F, Perry IJ, Lutomski J, et al. Temporal trends in misclassification patterns of measured and self-report based body mass index categories: findings from three population surveys in Ireland. BMC Public Health 2010, 10, 560.

[14] Stommel $M$ and Osier N. Temporal changes in bias of body mass index scores based on self-reported height and weight. Int $\mathcal{F}$ Obes 2013, 37, 461-467.

[15] Ikeda N. Validity of self-reports of height and weight among the general adult population in Japan: findings from National Household Surveys, 1986. PLoS One 2016, 11, e0148297

[16] Lu S, Su J, Xiang Q, et al. Accuracy of self-reported height, weight, and waist circumference in a general adult Chinese population. Popul Health Metr 2016, 14, 30-016-0099-8. eCollection.

[17] Anai A, Ueda K, Harada K, et al. Determinant factors of the difference between self-reported weight and measured weight among Japanese. Environ Health Prev Med 2015, 20, 447-454

[18] Celis-Morales C, Livingstone KM, Woolhead C, et al. How reliable is internet-based self-reported identity, socio-demographic and obesity measures in European adults? Genes Nutr 2015, 10, 28.

[19] Niedzwiedzka E, Dlugosz A and Wadolowska L. Validity of self-reported height and weight in elderly Poles. Nutr Res Pract 2015, 9, 319-327.

[20] Skeie G, Mode N, Henningsen M, et al. Validity of selfreported body mass index among middle-aged participants in the Norwegian Women and Cancer study. Clin Epidemiol $2015,7,313-323$

[21] Wright FL, Green J, Reeves G, et al. Validity over time of selfreported anthropometric variables during follow-up of a large cohort of UK women. BMC Med Res Methodol 2015, 15, 81.

[22] Hsiao H, Weaver D, Hsiao J, et al. Comparison of measured and self-reported anthropometric information among firefighters: implications and applications. Ergonomics 2014, 57, 1886-1897.

[23] Magnusson K, Haugen IK, Osteras N, et al. The validity of self-reported body mass index in a population-based osteoarthritis study. BMC Musculoskelet Disord 2014, 15, 442.

[24] Murphy G, Snetselaar L, Myers E, et al. Self-reported health parameters compared with clinician measurements: methods in practice-based research. $f$ Public Health Manag Pract 2014, 20, 513-522.

[25] Poston WS, Jitnarin N, Haddock CK, et al. Accuracy of selfreported weight, height and BMI in US firefighters. Occup Med 2014, 64, 246-254.

[26] Pursey K, Burrows TL, Stanwell P, et al. How accurate is web-based self-reported height, weight, and body mass index in young adults? $\mathcal{F}$ Med Internet Res 2014, 16, e4.

[27] Tolonen H, Koponen P, Mindell JS, et al. Under-estimation of obesity, hypertension and high cholesterol by self- 
reported data: comparison of self-reported information and objective measures from health examination surveys. Eur $\mathcal{F}$ Public Health 2014, 24, 941-948.

[28] Tsai EW, Perng W, Mora-Plazas M, et al. Accuracy of selfreported weight and height in women from Bogota, Colombia. Ann Hum Biol 2014, 41, 473-476.

[29] Xie YJ, Ho SC, Liu ZM, et al. Comparisons of measured and self-reported anthropometric variables and blood pressure in a sample of Hong Kong female nurses. PLoS One 2014, 9, e107233.

[30] Yoon K, Jang SN, Chun H, et al. Self-reported anthropometric information cannot vouch for the accurate assessment of obesity prevalence in populations of middle-aged and older Korean individuals. Arch Gerontol Geriatr 2014 59, 584-592.

[31] Bonn SE, Trolle Lagerros $\mathrm{Y}$ and Balter K. How valid are web-based self-reports of weight? $\mathcal{F}$ Med Internet Res 2013, $15, \mathrm{e} 52$.

[32] Lassale C, Peneau S, Touvier M, et al. Validity of web-based self-reported weight and height: results of the NutrinetSante study. 7 Med Internet Res 2013, 15, e152.

[33] May AM, Barnes DR, Forouhi NG, et al. Prediction of measured weight from self-reported weight was not improved after stratification by body mass index. Obesity 2013, 21, E137-142.

[34] Pasalich M, Lee AH, Burke L, et al. Accuracy of self-reported anthropometric measures in older Australian adults. Australas F Ageing 2014, 33, E27-32.

[35] Yoong SL, Carey ML, D'Este C, et al. Agreement between self-reported and measured weight and height collected in general practice patients: a prospective study. BMC Med Res Methodol 2013, 13, 38.

[36] Großschädl F, Haditsch B and Stronegger WJ. Validity of selfreported weight and height in Austrian adults: sociodemographic determinants and consequences for the classification of BMI categories. Public Health Nutr 2012, 15, 20-27.

[37] Lin CJ, DeRoo LA, Jacobs SR, et al. Accuracy and reliability of self-reported weight and height in the Sister Study. Public Health Nutr 2012, 15, 989-999.

[38] Wen M and Kowaleski-Jones L. Sex and ethnic differences in validity of self-reported adult height, weight and body mass index. Ethn Dis 2012, 22, 72-78.

[39] Yong V and Saito Y. How accurate are self-reported height, weight, and BMI among community-dwelling elderly Japanese? Evidence from a national population-based study. Geriatr Gerontol Int 2012, 12, 247-256.

[40] Bes-Rastrollo M, Sabate J, Jaceldo-Siegl K, et al. Validation of self-reported anthropometrics in the Adventist Health Study 2. BMC Public Health 2011, 11, 213.

[41] Dijkshoorn H, Ujcic-Voortman JK, Viet L, et al. Ethnic variation in validity of the estimated obesity prevalence using self-reported weight and height measurements. BMC Public Health 2011, 11, 408

[42] Griebeler ML, Levis S, Beringer LM, et al. Self-reported versus measured height and weight in Hispanic and nonHispanic menopausal women. $f$ Womens Health 2011, 20, 599-604.

[43] Hayes AJ, Clarke PM and Lung TW. Change in bias in self-reported body mass index in Australia between 1995 and 2008 and the evaluation of correction equations. Popul Health Metr 2011, 9, 53.

[44] Isidoro B, Lope V, Pedraz-Pingarron C, et al. Validation of obesity based on self-reported data in Spanish women participants in breast cancer screening programmes. BMC Public Health 2011,11, 960.

[45] Krul AJ, Daanen HA and Choi H. Self-reported and measured weight, height and body mass index (BMI) in Italy, the Netherlands and North America. Eur f Public Health $2011,21,414-419$.
[46] Lee DH, Shin A, Kim J, et al. Validity of self-reported height and weight in a Korean population. F Epidemiol 2011, 21, $30-36$

[47] Lois K, Kumar S, Williams N, et al. Can self-reported height and weight be relied upon?. Occup Med 2011, 61, 590-592.

[48] Ng SP, Korda R, Clements M, et al.Validity of self-reported height and weight and derived body mass index in middleaged and elderly individuals in Australia. Aust N Z F Public Health 2011, 35, 557-563.

[49] Park JY, Mitrou PN, Keogh RH, et al. Effects of body size and sociodemographic characteristics on differences between self-reported and measured anthropometric data in middle-aged men and women: the EPIC-Norfolk study. Eur f Clin Nutr 2011,65, 357-367.

[50] Shields M, Connor Gorber S, Janssen I, et al. Bias in selfreported estimates of obesity in Canadian health surveys: an update on correction equations for adults. Health Rep 2011 $22,35-45$.

[51] Van Valkengoed IG, Nicolaou M and Stronks K. Ethnic differences in discrepancies between self-reported and measured weight, height and body mass index. Eur $\mathcal{F}$ Public Health 2011, 21, 420-423.

[52] Burton NW, Brown W and Dobson A. Accuracy of body mass index estimated from self-reported height and weight in mid-aged Australian women. Aust $N Z \mathcal{F}$ Public Health 2010, 34, 620-623.

[53] Dahl AK, Hassing LB, Fransson EI, et al. Agreement between self-reported and measured height, weight and body mass index in old age: a longitudinal study with 20 years of follow-up. Age Ageing 2010,39, 445-451.

[54] Fillenbaum GG, Kuchibhatla MN, Whitson HE, et al. Accuracy of self-reported height and weight in a community-based sample of older African Americans and whites. 7 Gerontol A Biol Sci Med Sci 2010, 65, 1123-1129.

[55] Lucca A and Moura EC. Validity and reliability of selfreported weight, height and body mass index from telephone interviews. Cad Saude Publica 2010, 26, 110-122.

[56] Meng H, He XZ and Dixon D. Self-reported versus measured height and weight in the health and retirement study. f Am Geriatr Soc 2010, 58, 412-413.

[57] Ahluwalia IB, Tessaro I, Rye S, et al. Self-reported and clinical measurement of three chronic disease risks among lowincome women in West Virginia. FWomens Health 2009, 18, 1857-1862.

[58] Merrill RM and Richardson JS. Validity of self-reported height, weight, and body mass index: findings from the National Health and Nutrition Examination Survey, 20012006. Prev Chronic Dis 2009, 6, A121.

[59] Oliveira A, Ramos E, Lopes C, et al. Self-reporting weight and height: misclassification effect on the risk estimates for acute myocardial infarction. Eur f Public Health 2009, 19, 548-553.

[60] Stommel M and Schoenborn CA. Accuracy and usefulness of BMI measures based on self-reported weight and height: findings from the NHANES \& NHIS 2001-2006. BMC Public Health 2009, 9, 421

[61] Danubio ME, Miranda G, Vinciguerra MG, et al. Comparison of self-reported and measured height and weight: implications for obesity research among young adults. Econ Hum Biol 2008, 6, 181-190.

[62] Dekkers JC, van Wier MF, Hendriksen IJ, et al. Accuracy of self-reported body weight, height and waist circumference in a Dutch overweight working population. BMC Med Res Methodol 2008, 8, 69.

[63] Elgar FJ and Stewart JM. Validity of self-report screening for overweight and obesity. Evidence from the Canadian Community Health Survey. Can F Public Health 2008, 99, 423-427. 
[64] Jeffery RW, Finch EA, Linde JA, et al. Does clinical depression affect the accuracy of self-reported height and weight in obese women?. Obesity 2008, 16, 473-475.

[65] Larsen JK, Ouwens M, Engels RC, et al. Validity of selfreported weight and height and predictors of weight bias in female college students. Appetite 2008, 50, 386-389.

[66] Paradis AM, Perusse L, Godin G, et al. Validity of a selfreported measure of familial history of obesity. Nutr $\mathcal{F} 2008$, $7,27$.

[67] Sahyoun NR, Maynard LM, Zhang XL, et al. Factors associated with errors in self-reported height and weight in older adults. F Nutr Health Aging 2008, 12, 108-115.

[68] Brunner Huber LR. Validity of self-reported height and weight in women of reproductive age. Matern Child Health $\mathcal{F}$ 2007, 11, 137-144.
[69] McAdams MA, Van Dam RM and Hu FB. Comparison of self-reported and measured BMI as correlates of disease markers in US adults. Obesity 2007, 15, 188-196.

[70] Nyholm M, Gullberg B, Merlo J, et al. The validity of obesity based on self-reported weight and height: Implications for population studies. Obesity 2007, 15, 197-208.

[71] White MA, Masheb RM, Burke-Martindale C, et al. Accuracy of self-reported weight among bariatric surgery candidates: the influence of race and weight cycling. Obesity 2007, $15,2761-2768$

[72] Taylor AW, Dal Grande E, Gill TK, et al. How valid are self-reported height and weight? A comparison between CATI self-report and clinic measurements using a large cohort study. Aust $N Z$ f Public Health 2006, 30, 238-246. 